\title{
Toward Making Epidemiologic Data More Useful for Quantitative Risk Assessment
}

\author{
Kenny S. Crump ${ }^{*, 1}$ and Bruce Allen ${ }^{2}$ \\ ${ }^{1}$ Louisiana Tech University, Ruston, LA, USA \\ ${ }^{2}$ Bruce Allen Consulting, Chapel Hill, NC, USA
}

\begin{abstract}
This paper discusses issues faced in using epidemiologic data to develop quantitative estimates of risk from specified patterns of exposure to a toxicant. We focus on use of data from cohort studies with binary endpoints (occurrence or non-occurrence of disease). Relative advantages of Cox regression and Poisson regression are presented. A general form of exposure metric is presented, and criteria for selecting an appropriate metric are discussed. Advantages and disadvantages of various dose-response models are discussed. It is argued that, unless low-dose linearity of the dose response can be ruled out on non-statistical grounds, then bounds for low-dose risk should incorporate low-dose linearity; a sequential procedure for computing such bounds is illustrated. Limitations in exposure data and their impact on risk assessments are discussed. Issues arising when using meta-analytic techniques to combine data from multiple epidemiologic studies are discussed. Limitations in risk assessments resulting from reliance upon published results alone are described. Methods for converting from measures of risk used in epidemiologic studies (e.g., relative risk) to measures appropriate for a risk assessment (e.g., additional lifetime probability of disease occurrence resulting from a specific exposure pattern) are described in detail. Several examples from the asbestos epidemiologic literature are presented to illustrate these issues.
\end{abstract}

Keywords: Epidemiologic data, cohort study, cox analysis, poisson analysis, relative risk model, exposure metric, low-dose risk, life table analysis.

\section{INTRODUCTION}

Usually the primary goal of an epidemiologic study is to determine if a causal link exists between exposure to a potentially toxic substance and disease in a defined population. If such a link is established the study may be subsequently applied in a risk assessment to quantify the relationship between exposure and risk to health in a target population that has demographic characteristics and exposure patterns that differ from those in the epidemiologic study. If multiple epidemiologic studies are available, the risk assessment should account for the totality of the information, perhaps by formally combining of data from multiple studies in a meta analysis. Measures of risk most often used in epidemiologic studies, such as relative risk, are normally not adequate for a risk assessment, which requires some measure of added risk.

Thus the analyses needed to support a quantitative risk assessment are usually different than, and often more extensive than, the analyses needed to support the initial goals of an epidemiologic study. Similarly, the data needs of the risk assessment may be more stringent. Whereas a link between exposure and health risk can perhaps be established using exposure surrogates such as duration of exposure, more specific exposure measures are needed to support a risk assessment.

\footnotetext{
*Address correspondence to this author at the Louisiana Tech, P.O. Box 10348, Ruston, LA 71272-0046, USA; Tel: 318-278-9426; Fax: 318-2572182; E-mail: KennyCrump@email.com
}

In this paper we discuss issues concerning the use of epidemiologic data to develop quantitative estimates of the lifetime risk of harm from given patterns of exposure to a toxic substance. Particular emphasis is given to estimating low-dose risk which might be useful in setting exposure standards. Issues addressed include selection of a statistical procedure for analyzing the epidemiologic data, selection of a dose-response model to use in the statistical procedure, converting results obtained using such models to estimates of additional lifetime risk from specified exposure patterns, and special issues related to estimating low-dose risk. These issues are illustrated using examples from the asbestos epidemiologic literature. (However, we caution that these examples are presented for pedagogical purposes only and are not intended for use in regulation of asbestos.)

If the response is a binary variable (presence or nonpresence of a particular disease) a risk assessment usually estimates the additional probability of disease. If the response is a continuous variable (e.g., IQ) the outcome of the risk assessment may be a summary measure of that continuous response (e.g., the mean change in IQ from a given pattern of exposure). Alternatively, a continuous response can be converted into a binary one (e.g., the additional proportion of a population with an IQ below a certain cutoff). Here we focus on quantifying the additional lifetime probability of a binary response from given exposure patterns, although much of the discussion will apply to other measures of risk. We also focus on use of data from cohort studies.

Despite the greater data needs of the risk assessment, risk assessments are often based only on published summaries of 
results from the epidemiologic investigations. This paper includes a discussion of methods for dealing with published data and gives examples of limitations of risk assessments due to lack of access to the unsummarized underlying data from epidemiologic studies.

\section{SELECTION OF A STATISTICAL METHOD}

Cox regression [1] and Poisson regression [2] are commonly used statistical methods for analyzing cohort data. In Poisson regression the person-years of observation are divided into cells defined by non-overlapping ranges of explanatory variables (e.g., gender, age, year, and exposure-related variables). A representative value for each variable is assigned to each cell (e.g., the average value in the cell). A regression involving these variables is conducted using the assumption that the number of cases occurring in each cell is Poisson distributed, with the mean response in a cell predicted by the regression model. Baseline rates (no exposure) can be estimated from the cohort data, or else external rates (e.g., national) can be incorporated into the regression model.

In Cox regression the data are categorized into risk sets defined by the individuals remaining in the study at the time of a death from the cause of interest. Individual responses are only compared at the same time so the time variable is completely controlled in the analysis. For diseases that vary more strongly with age than with calendar year, age is a more appropriate definition of the time variable than calendar time for defining risk sets. Calendar time can be controlled, if necessary, by being included as a variable in the regression model. In Cox regression only the risk relative to the baseline rate is estimated and the baseline rate itself is not estimated.

One advantage of Cox regression is that, unlike Poisson regression, it does not require arbitrarily dividing the data into cells. However, any dependence of the results of a Poisson regression on the cell definitions can be minimized by making the subdivisions that define the cells sufficiently fine. In a large cohort, computation time can become an issue with Cox regression, especially if some of the explanatory variables (e.g., exposure variables) are time-dependent. Whereas Cox regression is limited to regression models for relative risk, Poisson regression is more flexible and can be used with a much wider class of risk models. This can be an important advantage, especially when conducting a risk assessment rather than testing for a dose-rate effect. Also, the ability of Poisson regression to incorporate external rates into the modeling can be useful, as illustrated in the following example.

In addition to Cox and Poisson regression, a third alternative is to compute the full likelihood of the data in which each individual makes an independent contribution, and to estimate parameters by maximizing the resulting log-likelihood. Examples include risk assessments for leukemia from exposure to benzene [3, 4] and for mesothelioma from exposure to asbestos [5].

\section{EXAMPLE: COMPARISON OF POISSON AND COX REGRESSION IN MODELING LUNG CANCER IN AN ASBESTOS-EXPOSED COHORT}

A cohort of 6,358 men employed at a crocidolite mine and mill in Wittenoom, Australia between 1943 and 1946 experienced an excess of mortality from mesothelioma and lung cancer [6-12]. In a follow-up of the cohort through the year 2000 [5] 302 lung cancer deaths were recorded. The data base contains, in addition to cause of death information, date of birth, date last known to be alive, date of death, dates of beginning and ending of employment and estimates of the average intensity of exposure to crocidolite fibers (fibers per milliliter of air) during employment.

The U.S. EPA model for asbestos-related lung cancer [13] assumes that the relative risk of lung cancer mortality is given by

$R R=1+K_{L} C_{10}$

where $\mathrm{C}_{10}$ is cumulative exposure to asbestos (fiberyears $/ \mathrm{ml}$ ) lagged 10 years (i.e., exposures in the most recent ten years are not counted) and $\mathrm{K}_{\mathrm{L}}$ is the estimated potency parameter for lung cancer. Since this is a relative risk model it can be fit using either Cox regression or Poisson regression.

An expanded version of (Eq. 1),

$R R=\alpha\left(1+K_{L} C_{10}\right)$

includes a background parameter, $\alpha$, which allows for the possibility that the background mortality rates appropriate for the cohort differed from those of Australian men in general. This expanded model is not needed with Cox regression, which estimates risk relative to the (studyspecific) baseline.

Berman and Crump [5] fit both models to the Wittenoom data using Poisson regression. Age- and calendar-year-specific lung cancer mortality rates for Australian men were used for background rates. To compare with these results, we fit Eq. 1 to the Wittenoom data using Cox regression. The estimate of $\mathrm{K}_{\mathrm{L}}$ from the Cox regression agreed very closely with the estimate from the Poisson regression analysis in which $\alpha$ was estimated (Table $\mathbf{1}$ ). However, the Poisson regression provided information not available from the Cox regression about the relationship between the lung cancer rate in the general population and the baseline rate estimated for the cohort. The Poisson regression indicated that the baseline rate in the cohort was almost three times that of men in the general Australian population. This factor seems too large to be due to differences in smoking habits. Moreover, the evidence for a dose response in lung cancer with increasing exposure was somewhat limited, although statistically significant, even though exposures were very high in this cohort [5]. On the other hand, if the baseline rate is restricted to be the same as in the general Australian male population $(\alpha=1)$, the estimate of $\mathrm{K}_{\mathrm{L}}$ is about ten times higher than when $\alpha$ is estimated (Table 1). The model defined by (Eq. 2) provides a reasonable fit to the data when $\alpha$ is estimated (Fig. 1, solid line) but under-predicts the response at low exposures and over-predicts at high exposures when $\alpha=1$ (Fig. 1, dotted line).

This observed pattern may be partially due to misclassification of exposure along with higher levels of smoking in the cohort. This uncertainty, coupled with the large difference between the estimate of $\mathrm{K}_{\mathrm{L}}$ with $\alpha$ estimated and with $\alpha=1$ translates into an uncertainty in the estimate of $\mathrm{K}_{\mathrm{L}}$. Berman and Crump [5] dealt with this uncertainty in an $a d$ hoc fashion by limiting $\alpha$ to be no larger than 2.0. 
Table 1. Results from Applying the U.S. EPA Lung Cancer Model (Eqs. 1 and 2) to Data on a Cohort of Workers Exposed to Crocidolite Asbestos at Wittenoom, Australia

\begin{tabular}{|c|c|c|c|c|}
\hline Type of Analysis & $\boldsymbol{\alpha}$ & $\mathbf{( 9 0 \%} \mathbf{C I})$ & $\mathbf{K}_{\mathbf{L}}$ & $\mathbf{( 9 0 \%} \mathbf{C I})$ \\
\hline \hline Cox (this paper) & --- & --- & 0.0044 & $(0.0019,0.0076)$ \\
\hline Poisson [5] & 2.81 & $(2.49,3.14)$ & 0.0042 & $(0.0016,0.0076)$ \\
\hline Poisson [5] & 1(fixed) & --- & 0.043 & $(0.034,0.053)$ \\
\hline
\end{tabular}

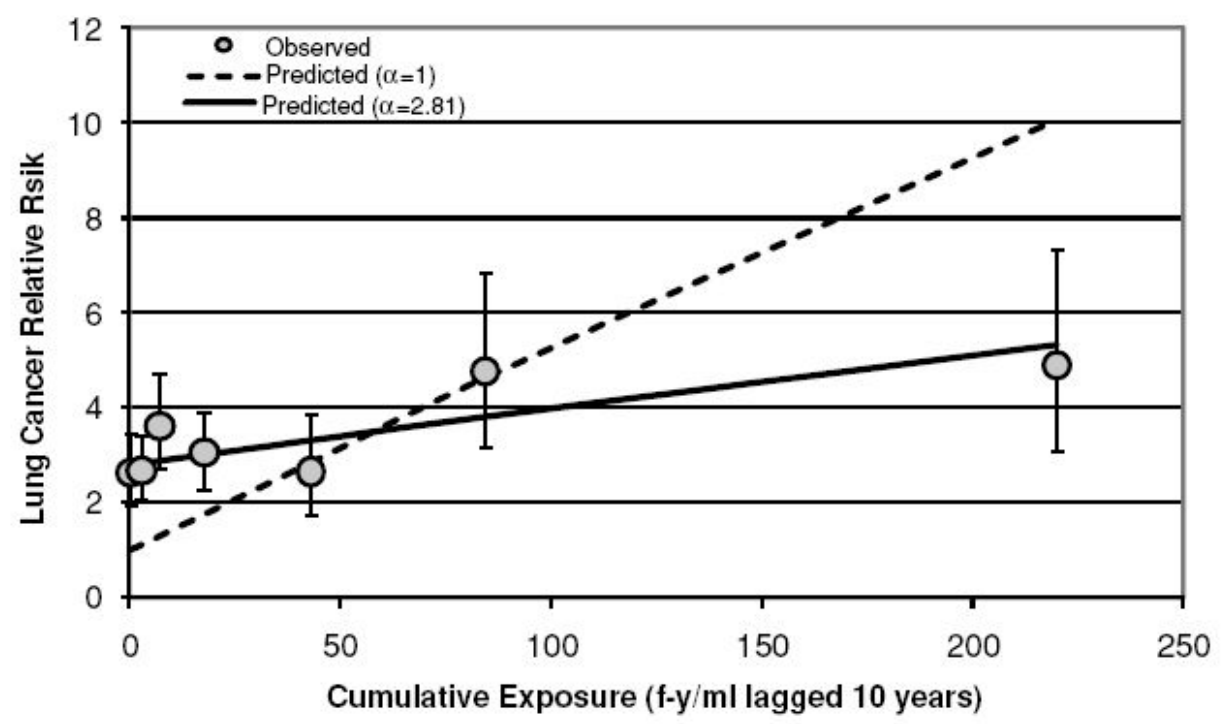

Numbers of lung cancers by Increasing cumulative exposure are 50, 65, 53, 57, 25, 29, 23.

Fig. (1). Fit of U.S. EPA lung cancer model (Eq. 2) to data on a cohort of workers exposed to crocidolite asbestos at Wittenoom, Australia. Vertical bars indicate $90 \%$ confidence intervals [5].

This example illustrates that a Poisson regression can provide information that cannot be deduced from a Cox regression. It also illustrates that if a background parameter (e.g., $\alpha$ ) is included in a Poisson regression, external background mortality rates can be included in the model to control for age, calendar time, race and gender without requiring the assumption that background rates in the cohort be identical with the external rates. Controlling for these covariates in this way can provide good control for these covariates, and at the same time result in a much simpler model with fewer explanatory variables to estimate. This type of regression analysis can be particularly useful in controlling statistical variability when the cohort study is of modest size.

\section{SELECTION OF AN EXPOSURE METRIC}

Developing a dose-response model requires specifying one or more summary measures of exposure which we will refer to as "exposure metrics." Ideally we would like to have a single summary metric that contains all of the information needed to inform dose response, i.e., a metric such that any additional exposure information would be superfluous with respect to characterizing the effect of exposure. Unfortunately, there is no known way to identify such a metric (assuming that such a metric exists).

Metrics that are commonly applied in epidemiologic studies include cumulative exposure, average exposure, peak exposure, and duration of exposure. Often several such metrics may be used to determine which ones show stronger statistical associations between exposure and a health outcome. Such exploratory analyses can be particularly useful in identifying an exposure effect when, as is often the case, exposure information is limited or of poor quality,

However, requirements for an exposure metric in risk assessment are more stringent. For a metric to be useful in risk assessment, it should account for both the duration and intensity of exposure. For example, average exposure is often calculated by averaging only over the period of exposure. Thus working for only one day exposed to $1 \mathrm{ppm}$ and working for 40 years continuously exposed to $1 \mathrm{ppm}$ would both correspond to an average exposure of $1 \mathrm{ppm}$, although they are unlikely to present the same risk. Other metrics that don't integrate duration and intensity of exposure include duration of exposure and peak exposure. Although such metrics generally are not directly useful in risk assessment models, results from their use could be helpful in choosing an appropriate exposure metric.

A fairly general form for an exposure metric that integrates duration and intensity can be defined as

$X(t)=\int_{0}^{t} g[E(u)] h(t, u) d u$,

where $\mathrm{X}(\mathrm{t})$ is the value of the exposure metric at time $\mathrm{t}, \mathrm{E}(\mathrm{u})$ is exposure intensity at time $\mathrm{u}, \mathrm{g}$ allows for a non-linear effect of exposure intensity, and $\mathrm{h}(\mathrm{t}, \mathrm{u})$ is a weighting 
function that gives the effect at time $\mathrm{t}$ relative to the exposure at time u. Several common metrics are special cases of Eq. 3. If $\mathrm{g}(\mathrm{E})=\mathrm{E}$ and $\mathrm{h}(\mathrm{t}, \mathrm{u})=1$, then

$X(t)=\int_{0}^{t} E(u) d u$,

which is cumulative exposure. If $g(E)=E$ and $\mathrm{h}(\mathrm{u}, \mathrm{t})=1 / \mathrm{t}$ then

$X(t)=\frac{\left[\int_{0}^{t} E(u) d u\right]}{t}$

which is average exposure between 0 and $\mathrm{t}$. If $\mathrm{g}(\mathrm{E})=\mathrm{E}$ and

$h(t, u)= \begin{cases}0 & \text { if } t-u \leq L \\ (t-u)^{2} & \text { if } t-u>L\end{cases}$

then

$X(t)=\int_{0}^{t-L} E(t)(t-u)^{2} d u$.

If $\mathrm{L}=10, \mathrm{E}(\mathrm{t})=\mathrm{E}$ (constant) for $\mathrm{t}$ between 0 and Dur (exposure duration) and zero otherwise, then this becomes the exposure metric used in the U.S. EPA [13] model for mesothelioma from asbestos exposure,

$X(t)=\left\{\begin{array}{cr}0 & \text { if } t \leq 10 \\ (t-10)^{3} E & \text { if } 10<t \leq 10+\text { Dur } \\ {\left[(t-10)^{3}-(t-10-\text { Dur })^{3}\right] E} & \text { if } 10+\text { Dur }<t .\end{array}\right.$ (Eq)

Alternatively, Eq. 7 can be viewed as a more general form of Eq. 8 that applies to non-constant exposure. With the metrics defined by Eqs. 7 and 8, the effect of exposure in a given time increment increases indefinitely as the square of elapsed time from when the exposure occurred. To model an exposure effect that reaches a peak after the exposure and then decreases to zero, one could use a weighting function of the form $\mathrm{h}(\mathrm{t}, \mathrm{u})=(\mathrm{t}-\mathrm{u})^{\mathrm{K}} \exp [-\alpha(\mathrm{t}-\mathrm{u})]$ [14]. To model an effect of peak exposure (exposure that has no effect until the exposure intensity exceeds a minimum value, $\mathrm{E}_{\min }$ ), one could define, e.g.,

$g(E)= \begin{cases}0 & \text { if } E \leq E_{\min } \\ \left(E-E_{\min }\right)^{k} & \text { if } E_{\min }<E .\end{cases}$

Alternatively, g could represent a pharmacokinetic model that relates external exposure to exposure to some internal organ.

The effect of simultaneous exposure to multiple toxicants can be accounted for in a risk assessment by assigning an exposure metric for each toxicant and incorporating these metrics in a single dose-response model. A risk assessment for exposure to asbestos fibers that included a number of epidemiologic studies, each with a unique mix of fiber types and distribution of fiber sizes, illustrates such a case [15]. In this risk assessment, fibers were placed in one of several categories defined by length, width and type (chrysotile or amphibole), and each category of fibers was treated as a unique toxicant with its own exposure metric.

\section{CHOICE OF DOSE-RESPONSE MODEL: GENERAL CONSIDERATIONS}

A dose-response model relates an exposure metric, $\mathrm{X}$, to some measure of risk such as relative risk. The term "model" is often used loosely to refer to the statistical fitting method, as in a "Poisson model" or a "Cox model." However, many different dose-response models can be applied using either Cox or Poisson regression techniques, and, as we have already noted, the same dose-response model can often be fit with either type of regression.

Exposures for which risk estimates are needed for setting exposure standards will generally require extrapolation below the range of exposures for which exposure-related adverse responses were identified in an epidemiologic study. In these cases the low-dose properties of the dose-response model chosen will be critically important. The choice of model should take account of the biological plausibility of the selected dose response, particularly in the low-dose region. This is much less of a concern in an analysis that only seeks to determine whether or not a dose-related effect is present.

General low-dose curve shapes that have some biological plausibility range from linear to sub-linear, which includes a threshold curve shape - the most extreme version of sublinearity. A linear low-dose curve shape refers to a dose response that has a positive, finite slope at zero dose; a sublinear (sometimes referred to as non-linear) low-dose curve shape refers to a dose response that has a slope of zero at zero dose (e.g., dose $\mathrm{K}^{\mathrm{K}}, \mathrm{K}>1$ ). "Threshold" is a special case of sub-linearity that refers to a dose response whose slope remains zero over a dose interval ranging from zero dose up to some positive dose termed the threshold dose. A supralinear low-dose curve shape refers to a dose response that has an infinite slope at zero dose (e.g., $\operatorname{dose}^{\mathrm{K}}, 0<\mathrm{K}<1$ ). Such dose responses are generally thought to be biologically implausible.

Any set of dose-response data is statistically compatible with both threshold and low-dose linear curve shapes. Fig. (2) provides a graphical illustration of this point using a hypothetical data set with five dose groups each containing 50 subjects. There is no response in the control group or at the four lowest doses, but an increased response at the highest dose. As the figure shows, these data are well fit both by a dose-response model that is linear up through the four lowest doses (solid line) and one that has a threshold at a dose of 4 (dotted line). In fact it is true of both the low-dose linear model and the threshold model that the observed response at each dose is the most likely response predicted by the model. This illustrates a general result that does not depend upon the details of this example: every set of doseresponse data is compatible with both low-dose linear and threshold models.

Statistical methods for estimating thresholds have been proposed (e.g., [16]) and such methods have been applied to epidemiologic data ( e.g., [17]). These methods can be used to test whether data are consistent with a zero threshold (i.e., no threshold) or, equivalently, whether a statistical confidence interval for the threshold includes zero. Such methods employ a specific functional form for the dose response, usually the form shown in Fig. (2), which assumes that risk increases linearly with dose for doses that exceed the threshold. Applying this model to the data in Fig. (2) (dotted line in Fig. 2) results in a 95\% statistical lower bound of 3.5 for the threshold. Likewise the hypothesis that there is no threshold (threshold $=0$ ) is firmly rejected $(\mathrm{p}=0.0002)$. Nevertheless, as Fig. (2) shows, non-threshold dose responses can fit these data quite adequately. This demonstrates that these statistical methods are highly model dependent. They provide statistical evidence for a threshold 


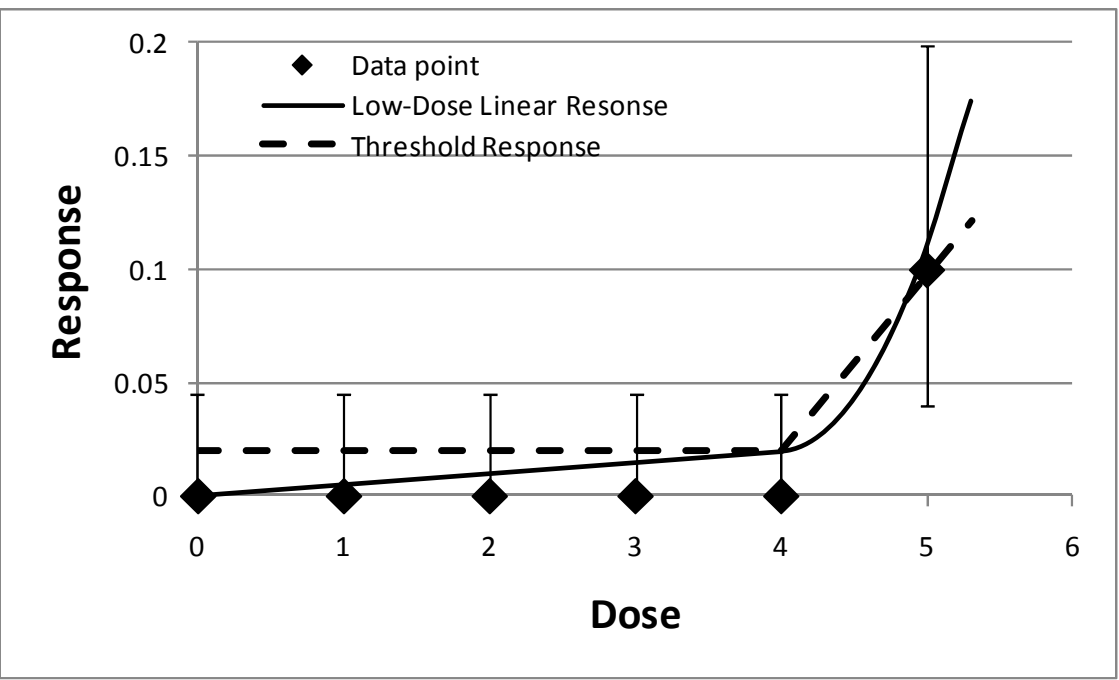

Fig. (2). Illustration that any set of dose-response data is statistically compatible with both threshold and low-dose linear curve shapes. Vertical bars indicate $90 \%$ confidence intervals.

only if one is willing to accept that the underlying dose response has the assumed form - one that increases linearly in dose for doses above the threshold. Since we never have such detailed knowledge about the shape of the true dose response, statistical tests for the existence of a threshold are apt to be misleading.

This example illustrates that even if a threshold exists, it will not be possible to bound its estimate away from zero without making detailed and generally unverifiable assumptions about the shape of the dose response at doses exceeding the threshold. Since some amount of low-dose linearity is always consistent with the data, and supralinearity is implausible, unless low-dose linearity is ruled out on non-statistical grounds, conservative estimates of low-dose risk need to incorporate low-dose linearity. Statistical methods that can be useful in making such estimates are discussed in the next section.

\section{CHOICE OF DOSE RESPONSE MODEL: APPLICATION}

Dose-response models used in epidemiologic studies are often chosen out of convenience rather than with consideration of low-dose properties. One commonly used dose-response model is the log-linear model for relative risk,

$R R=\exp (\beta X)$

where $\mathrm{X}$ is the exposure metric and $\beta$ a parameter that gauges the potency of the toxic substance. This model can be conveniently applied using either Poisson or Cox regression implemented using one of a number of standard software programs, which can also accommodate additional explanatory variables such as age, gender, etc. With the dose response defined by Eq. $10, \log \mathrm{RR}$ is linear in dose. The $\log$-untransformed relative risk has a slope of $\beta$ at zero dose and consequently is linear at low dose, although it curves increasingly upward with increasing dose. The linear relative risk model,

$R R=1+\beta X$,

which is used less often than the log-linear model, has a slope of $\beta$ at all doses.
Epidemiological dose-response data frequently exhibit downward curvature and neither a log-linear nor a linear dose-response model provides an adequate fit. Several reasons have been suggested to explain this effect, including 1) mismeasurement of high exposures due to, for example, not accounting for respirator use by highly exposed individuals, 2) saturation of metabolic pathways at high exposures, 3) depletion of susceptible individuals at high exposures, and 4) bias resulting from the healthy worker survivor effect [18]. A dose response that is downward curving due to saturation of metabolic pathways could be linear if a more appropriate dose metric based on internal metabolite concentrations were used. The depletion of susceptible individuals at high exposures might occur if the susceptible individuals succumbed to other related effects, prematurely removing them from the risk pool for the endpoint of interest, or if they tended to leave employment or switch to jobs having lower exposures. None of these reasons argues that there is a downward curving relationship between the "right" exposure metric and the "true" response rate. Rather, each of those explanations merely suggest why there might be apparent downward curving relationships between either A) an exposure metric that is less than optimal because of measurement or relevance problems and a reasonable measure of response (reasons 1 and 2), or B) a reasonable exposure metric and a mismeasured response rate (reasons 3 and 4). Of course, more than one of these reasons may contribute to the apparent curve shape.

When a dose response curves downward, a better fit and stronger evidence of an exposure effect is often obtained by applying the log-linear model (Eq. 10) to log-transformed exposures, resulting in the model

$R R=\exp [\beta \operatorname{Ln}(X)]=X^{\beta}$.

If $\beta<1$ this model exhibits downward curvature, which allows it to fit downward curving dose-response data better than either a log-linear (Eq. 10) or linear (Eq. 11) doseresponse model. Even though this model can be useful in testing for an exposure effect, it may not be a good choice if the goal is to estimate low-dose risk. When $\beta<1$ this model is supra-linear at low dose (has an infinite slope at $\mathrm{X}=0$ ). 
Moreover, rather than predicting a relative risk of 1.0 at zero exposure $(X=0)$, as it should, this model predicts a relative risk of zero, which nonsensically implies that an unexposed individual is at infinitely less risk than one who is exposed.

If there are unexposed individuals in the cohort, $\operatorname{Ln}(\mathrm{X})$ cannot be calculated (although as just noted, the model (Eq. 12) predicts $R R=0$ in this case), and to allow the calculation to proceed when using standard statistical software, a small amount, c, is sometimes added to the exposure variable, resulting in the adjusted model

$R R=\exp \left(\beta \operatorname{Ln}(c+X)=(c+X)^{\beta}\right.$.

Although this model is linear at low dose, the relative risk is 1 at zero exposure $(\mathrm{X}=0)$ only if $\mathrm{c}=1$.

\section{METHODS FOR BOUNDING LOW-DOSE RISK}

As noted earlier, low-dose linearity can never by ruled out on statistical grounds. If low-dose linearity also cannot be ruled out on non-statistical grounds, upper bounds on low-dose risk should allow for low-dose linearity. If the linear dose-response model (Eq. 11) adequately describes the data then a fit of this model to the entire data set could reasonably be used to set an upper bound for low-dose risk.

When a linear model does not describe epidemiologic adequately, it is often because the dose response exhibits downward curvature. One approach for dealing with downward curving data is to apply a piecewise linear model composed of straight lines connected end to end, one for lower doses that has a higher slope and one for higher doses with a lower slope [18]. However, the high dose data where the dose response is clearly downward curving are unlikely to be informative about the low-dose slope and including these data in the fitting could bias the estimate of the lowdose slope. The same can be said for a model that uses logtransformed exposure (Eq. 13).

Here we suggest an alternative that involves estimating the low-dose slope from a fit only to data in a lower dose range. A decision must be made about which data to include in such an analysis and there is a trade-off with respect to selecting the cutoff for data inclusion (data with exposures below the cutoff are included; data with exposures above the cutoff are excluded). If the cutoff is too high, then one fails to eliminate the undesirable consequences of including data that are problematic (for one or more of the reasons cited above). If the cutoff is set too low, then the slope estimate will be statistically unstable because of too few observations.

To determine an appropriate cutoff, we suggest a sequential procedure. First a linear dose-response model (e.g., Eq. 11) is expanded by replacing the exposure metric $\mathrm{X}$ by $\mathrm{X}^{\mathrm{K}}$ and this expanded model is fit to the data set. If this model finds significant downward curvature (i.e., the estimate of $\mathrm{K}$ is statistically significantly less than 1.0 ), the data corresponding to the highest exposures are eliminated and the expanded model is refit to the remaining data. If the estimate of $\mathrm{K}$ is still significantly less than 1.0 this process is repeated until the estimate of $\mathrm{K}$ is not statistically less than 1.0. Then the remaining data are fit to the linear model (with $K=1$ ) and the resulting estimate of $\beta$ is used as the estimate of the low-dose slope.
The potential advantages of this approach can be seen in relation to the reasons cited above for apparent downward curvature in the relationship between exposure and response. If the exposures in the highly exposed groups are misclassified, e.g., due to respirator use, resulting in overestimation to some unknown degree, then the procedure described above removes the most problematic data from the analysis. If the misclassification of exposure is because of saturable metabolism, then focusing attention on the lower exposures corresponds to the exposure levels for which saturation is less of an issue, and for which linear estimation of metabolism is more appropriate. If the apparent downward curvature is due to loss (or shifting) of susceptible individuals, this again eliminates from consideration those dose groups for which the observed response would tend to be less representative. To the extent that the healthy worker effect is more pronounced or problematic for highly exposed individuals (e.g., because one has to be healthier in general to tolerate those higher exposures) then this procedure would also tend to circumvent that concern.

\section{EXAMPLE: ESTIMATING THE LOW-DOSE SLOPE OF THE MESOTHELIOMA DOSE RESPONSE FROM ASBESTOS EXPOSURE IN WITTENOOM, AUSTRA- LIA COHORT}

In the Wittenoom, Australia cohort described earlier, 222 deaths from pleural or peritoneal mesothelioma were recorded. (See [5,19] and references therein for further details.) Berman and Crump [5] fit the U.S. EPA mesothelioma dose-response model [13] to these data. For a fixed occupational exposure to $\mathrm{E}$ fibers $/ \mathrm{ml}$, for duration of Dur years, this model predicts a yearly mesothelioma mortality rate at $t$ years from first exposure of $K_{M} X(t)$, where the exposure metric $\mathrm{X}(\mathrm{t})$ is defined by Eq. 8, and $\mathrm{K}_{\mathrm{M}}$ is an estimated parameter. To test the assumption that the mortality rate is proportional to the exposure intensity $\mathrm{E}$, the model was expanded by replacing $E$ by $E^{K}$. In this expanded model the non-linearity parameter $\mathrm{K}$ was estimated as $\mathrm{K}=$ 0.47 and was significantly less than $1.0(\mathrm{p}<0.0001)$. The fit with $\mathrm{K}=0.47$ is reasonable, but with $\mathrm{K}=1$ the model underpredicts the mesothelioma mortality rate at low exposures and over-predicts at higher exposures (Fig. 3). Estimates of lifetime risk of mesothelioma death from lifetime exposure to 0.00023 fibers $/ \mathrm{ml}$ computed using methods described in the Appendix (details not provided) differ by a factor of 260 ( 0.00072 for $\mathrm{K}=1$ and 0.19 for $\mathrm{K}=0.47)$. With $\mathrm{K}=1$ and exposure intensity log-transformed (replacing $\mathrm{E}$ by $\operatorname{Ln}(1$ + E) in Eq. 8) the fit is improved over that of the linear model, as expected, and the corresponding risk estimate is 0.0065 which is 9 times larger than the estimate with $K=1$ and 29 times smaller than the estimate of $\mathrm{K}=0.47$ derived from the complete data set.

Table 2 shows the results of analyses after successively restricting the analysis to workers assigned lower exposures. As workers with higher exposures were successively eliminated, the exposure response remained significantly non-linear until only exposures $\leq 8 \mathrm{fibers} / \mathrm{ml}$ remained in the analysis. The potency estimate, $\mathrm{K}_{\mathrm{M}}$, increased almost fourfold (from 12.2 to 45.8) as the analysis went from no restrictions to including only exposures $\leq 8 \mathrm{fibers} / \mathrm{ml}$. Also, the width of the statistical confidence interval for $\mathrm{K}_{\mathrm{M}}$ increased as the number of data decreased. 


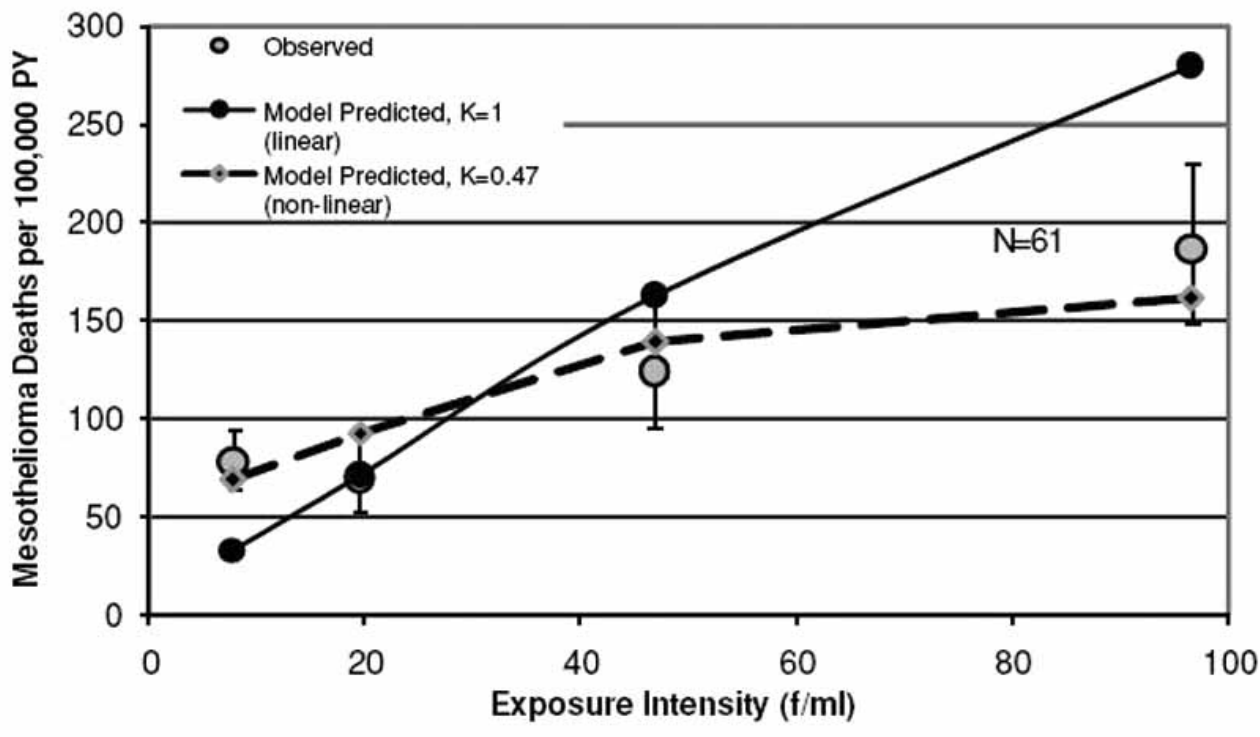

Numbers of mesotheliomas by Increasing exposure intensity are 79, 37, 45, 61.

Fig. (3). Observed mesothelioma death rate $v s$ exposure intensity compared to expected rates based on a linear dose-response model $(\mathrm{K}=1)$ and a non-linear model $(\mathrm{K}=0.47)$. Based on a cohort of workers exposed to crocidolite asbestos at Wittenoom, Australia. Vertical bars indicate $90 \%$ confidence intervals [5].

The non-linearity parameter $\mathrm{K}$ remained below 1.0 even when exposures were restricted to $\leq 5$ fibers $/ \mathrm{ml}$. The largest obtained estimate of $\mathrm{K}_{\mathrm{M}}$ was 110 , which occurred in the analysis restricted to exposures $\leq 1 \mathrm{fiber} / \mathrm{ml}$, which was the lowest exposure assigned to any worker. Misclassification of exposure may be partially responsible for consistent estimates of $\mathrm{K}<1$ and corresponding lack of fit of the linear model $(K=1)$, just as it may also have been responsible for the elevated estimate of $\alpha$ found earlier when the lung cancer model (Eq. 2) was applied to the Wittenoom data. Correcting for exposure errors or estimating the magnitude of their effect is very difficult without making strong assumptions or without more information than is available regarding exposures in this cohort. Absent additional information the results in Table 2 suggest an uncertainty of perhaps an order of magnitude in the value of $\mathrm{K}_{\mathrm{M}}$. Since the model predicts that risk at low exposures will vary linearly with $\mathrm{K}_{\mathrm{M}}$, a similar range of uncertainty will apply to the risk estimates as well.

\section{MODELS FOR DATA SHOWING A DOSE-RATE EFFECT}

By a dose-rate effect we mean a non-linearity in the exposure response whereby a given increase in intensity of exposure will cause a greater than proportional increase in risk. An example would be applying an exposure metric of the form of (Eq. 3) with $\mathrm{g}(\mathrm{E})=\mathrm{E}^{3}$. If a dose rate effect is present then use of a metric such as cumulative exposure will overestimate low-dose risk. A dose-rate effect is plausible for some health effects. For example, silica-induced silicosis - a type of pneumoconiosis that causes lung fibrosis

Table 2. Tests for Non-Linearity $(K<1)$ and Estimates of Low-Dose Slope $\left(K_{M}\right)$ in the U.S. EPA Mesothelioma Model Applied to Data on a Cohort of Workers Exposed to Crocidolite Asbestos at Wittenoom, Australia [5, 12]

\begin{tabular}{|c|c|c|c|c|c|c|c|}
\hline \multirow{2}{*}{ Including Exposures } & \multirow{2}{*}{$\begin{array}{l}\text { Number of } \\
\text { Subjects }\end{array}$} & \multirow{2}{*}{$\begin{array}{c}\text { Number of } \\
\text { Mesotheliomas }\end{array}$} & \multirow{2}{*}{$\begin{array}{c}\text { Nonlinear } \\
\text { Parameter K }\end{array}$} & \multirow{2}{*}{$\begin{array}{c}\begin{array}{c}\text { p-Value for } \\
\text { Non-Linearity }\end{array} \\
(\text { Test of } K=1)\end{array}$} & \multicolumn{2}{|c|}{$\begin{array}{l}\text { Estimate from Linear } \\
\quad \text { Model }(K=1)\end{array}$} & \multirow{2}{*}{$K_{M} /\left[K_{M}\right.$ Using All Data $]$} \\
\hline & & & & & $\mathbf{K}_{\mathbf{M}}$ & $(90 \% \mathrm{CI})$ & \\
\hline all & 6238 & 222 & 0.46 & $<0.0001$ & 12.2 & $(10.9,13.6)$ & 1 \\
\hline$\leq 100$ fibers $/ \mathrm{ml}$ & 5946 & 198 & 0.41 & $<0.0001$ & 12.8 & $(11.3,14.3)$ & 1.0 \\
\hline$\leq 70$ fibers $/ \mathrm{ml}$ & 5515 & 169 & 0.36 & $<0.0001$ & 14.6 & $(12.8,16.5)$ & 1.2 \\
\hline$\leq 50$ fibers $/ \mathrm{ml}$ & 5095 & 145 & 0.23 & $<0.0001$ & 15.1 & $(13.1,17.2)$ & 1.2 \\
\hline$\leq 20$ fibers $/ \mathrm{ml}$ & 4276 & 112 & 0.26 & $<0.0001$ & 21.1 & $(18,24.6)$ & 1.7 \\
\hline$\leq 10$ fibers $/ \mathrm{ml}$ & 2659 & 72 & 0.48 & 0.016 & 32.2 & $(26.4,38.9)$ & 2.6 \\
\hline$\leq 8$ fibers $/ \mathrm{ml}$ & 1058 & 27 & 0.52 & 0.12 & 45.8 & $(32.8,61.8)$ & 3.8 \\
\hline$\leq 5$ fibers $/ \mathrm{ml}$ & 934 & 19 & 0.31 & 0.044 & 43.9 & $(29.3,62.6)$ & 3.6 \\
\hline$\leq 1$ fibers $/ \mathrm{ml}$ & 381 & 6 & 1.00 & 1.0 & 110.0 & $(51.7,201.5)$ & 9.0 \\
\hline
\end{tabular}


and shortness of breath-may have a dose-rate effect due to the ability of high concentrations of silica in the lungs to interfere with clearance by alveolar macrophages.

A dose-rate effect can be demonstrated as recommended above for a downward curving dose response, except that the statistical test is for $K>1$ rather than $K<1$. The sequential procedure for identifying a low-exposure range consistent with linearity can also be used, but testing for $\mathrm{K}>1$.

Non-sequential approaches were applied by Hughes et al. [20] and Buchanan et al. [21] in modeling risk of silicosis (probability of a positive radiograph) among silica-exposed workers. Although these were studies of different cohorts and used different definitions of a positive radiograph, both studies found that the dose-response slope based on cumulative exposure was significantly higher among workers exposed to higher concentrations of silica $\left(>0.5 \mathrm{mg} / \mathrm{m}^{3}\right.$ by Hughes et al. and $>2 \mathrm{mg} / \mathrm{m}^{3}$ by Buchanan et al.) than to lower concentrations. Both analyses applied logistic models. However, whereas the Buchanan et al. model had a finite lowdose linear slope of $0.0035\left(\mathrm{~g}-\mathrm{h} / \mathrm{m}^{3}\right)^{-1}$, the Hughes et al. model was supra-linear at low dose.

\section{USE OF BIOLOGICALLY-BASED DOSE-RESPONSE (BBDR) MODELS IN ESTIMATING LOW-DOSE RISK}

BBDR models describe biological processes at the cellular and molecular level to link external exposure to an adverse response. A number of cancer risk assessments in the literature have applied the two-stage clonal expansion model of cancer [22] to epidemiologic data (e.g., [23,24]. This model accounts for mutation of normal cells to initiated cells, clonal expansion of initiated cells, and mutation of initiated cells to fully malignant cells. The incidence or mortality rate of cancer is expressed as a function of the mutation rates from normal to initiated cells and from initiated to fully malignant cells, and of the rates of cell division and death of initiated cells.

The clonal expansion model has been useful in generating and/or evaluating hypotheses concerning the mechanisms of action of toxicants (e.g., [24,25]). However, it is important to note that the model does not specify the functional forms for dose responses for the intermediate events leading to cancer. Consequently, use of the model does not obviate the need to make assumptions concerning those dose-response shapes; the dose responses used in the clonal expansion model must be developed empirically. In fact, use of the model may complicate the evaluation of the dose response because dose-response models must be assumed for each of the upstream rates that are assumed to be dose-related. Thus, the clonal expansion model appears to have few if any advantages over fully empirical models in quantifying low-dose risk. It seems likely that any BBDR model would have similar limitations [26]. The general paucity of human data on intermediate disease processes, and the uncertainty in human exposures in most epidemiologic studies would also limit any potential theoretical advantages of BBDR models in estimating low-dose risk from epidemiologic data.

\section{ISSUES IN QUANTIFYING EXPOSURES IN EPIDEMIOLOGIC STUDIES}

Risk estimates are equally dependent on measures of health effects and on measures of exposure. However exposure estimates are often much more uncertain than those for health effects. This is especially true when, as is usually the case, historical exposure data must be relied upon. Often the record of exposure levels at more distant times in the past is very spotty and the frequency and relevance of the measurements is less than desired. The methodology for measuring exposures may have evolved over the years, perhaps so much so that earlier measurements are not directly comparable to those obtained with newer and more accurate or otherwise improved techniques. Exposure reconstruction, both in terms of the measurement of concentrations as well as in relation to determining worker activities associated with specific jobs, is a subdiscipline of epidemiology in and of itself.

Errors and uncertainties in exposure estimates are particularly a problem in dose-response analysis. Exposure errors tend to attenuate a dose response and obliterate important details in the dose response. It was noted above that the high background risk of lung cancer suggested by the observed dose response in the Wittenoom, Australia cohort (Fig. 1) could be due to exposure misclassification. Exposure misclassification, even if unbiased, can cause a sublinear or threshold dose response to appear linear or even supralinear [27,28].

Although statistical methods have been developed to deal with exposure uncertainty [28], to be most useful, these methods require information that is seldom available in a cohort study. They also require assumptions about the distribution of true exposures vis-à-vis measured exposures that may be difficult to defend. Alternatively, methods based on expert judgment can be used to quantify uncertainty in exposure, e.g., by positing upper and lower ranges for exposures. These ranges can be incorporated into the risk assessment to obtain corresponding ranges for risk. Although such subjective methods are not optimal, they may be the best alternative in many cases. It should be remembered that subjective methods are often required to develop any estimates of exposures - for example, in the earliest times of follow-up when exposure information was limited or unavailable, or in areas of a plant where exposures were not routinely measured. Thus, subjective methods for quantifying uncertainty in exposures may be commensurate with the subjective nature of the exposure measures per se. A subjective approach for quantifying uncertainty in exposures in asbestos-exposed cohorts [15] is described below in the example to illustrate meta analyses.

\section{META ANALYSES}

When multiple epidemiologic studies are available, a meta analysis that combines data from different studies may be useful in representing the totality of the information available. If possible such an analysis should be based on the unprocessed data from each study, rather than from published summaries. Such analyses may require close collaboration from a number of different research groups. For example, authors of 10 occupational studies of cohorts exposed to silica collaborated in a meta analysis of silicainduced lung cancer using the unprocessed data from each cohort [17].

If the modeling approach used in the meta analysis incorporates background rates, as could be the case with Poisson regression, the model can estimate study-specific 
background rates to account for differences between the different cohorts in such factors as ethnicity and smoking that might affect background risk. Cohort-exposure interaction terms can be included to test for heterogeneity in the exposure effect present in each cohort [17]. If significant heterogeneity is identified, possible causes should be identified. If it appears to be caused by some feature in particular studies that makes them less appropriate for estimating risk in the target population (e.g., if a study was conducted among a predisposed population) then these studies perhaps should be removed from the analysis. If no reason for heterogeneity is identified, then it may be better for the dose response to be developed from all of the studies, and allow the heterogeneity to contribute to the uncertainty in the final result.

However, tests for heterogeneity are not necessarily useful for selecting a dose-response model. For example, Steenland et al. [17] found that when cumulative exposure lagged 15 years was used as the exposure metric significant heterogeneity was identified among the potencies estimated from the individual studies $(\mathrm{p}=0.02)$ but when $\log$ cumulative exposure lagged 15 years was used no significant heterogeneity was found $(\mathrm{p}=0.34)$. The finding of significant non-homogeneity suggests that other sources of variation are present that are not adequately controlled. Nevertheless, the untransformed metric provided a better fit to the complete data set $(\log$-likelihood $=21.4)$ than the $\log$ transformed metric $(\log$-likelihood $=18.8)$. The fact that the poorer fitting metric did not show significant nonhomogeneity could be due to the fact that it is relatively inflexible which makes it unable to detect the nonhomogeneity. The metric based on untransformed exposures fit better both with and without the interaction terms included. The better fitting metric should be preferred on statistical grounds despite the significant non-homogeneity.

Attempts to develop a meta analysis from published summaries can encounter numerous problems. The individual analyses may have been conducted in mutually inconsistent manners, e.g., using different exposure metrics or dose-response models. An example is presented next of a meta analysis that incorporates data from numerous sources.

\section{EXAMPLE: A META ANALYSIS OF ASBESTOS- RELATED CANCER RISK BASED ON PUBLISHED SUMMARIES THAT ADDRESSED FIBER SIZE AND MINERAL TYPE}

Estimates of the potency of asbestos for causing lung cancer or mesothelioma obtained from different occupational cohorts vary widely. It has been hypothesized that these disparities are due to differences in the types of fibers (chrysotile or amphibole) or in fiber dimensions present in the different study environments. Berman and Crump [15] conducted a meta analysis involving 15 asbestos-exposed cohorts to test whether taking differences in fiber size and type into account could help to reconcile the different asbestos potencies obtained from different studies.

The exposures in the individual studies were quantified by phase contrast microscopy (PCM), which weights all fibers longer than five microns equally and does not distinguish fiber types. Because these exposure measures were inadequate for evaluating the effect of fiber type and dimension, it was necessary to develop size- and typespecific exposure estimates for each environment. The fraction of asbestos exposure in each environment contributed by chrysotile was estimated using information available from the literature. Since these estimates were uncertain, uncertainty ranges for these fractions were developed, also using information available in the literature. Data were obtained from the published literature that provided size distributions of asbestos fibers in different occupational environments as measured by transmission electron microscopy (TEM). These size distributions were matched with the 15 epidemiologic studies based on being conducted in the same location or in a similar environment. These matched distributions were used to convert the original exposures in terms of fibers longer than five microns measured by PCM to estimates of exposures in different fiber size ranges as measured by TEM. Explicit factors were developed to account for different sources of uncertainty in the resulting exposure estimates, including uncertainty in a) PCM concentrations to which workers were exposed; b) converting from other measurement methods (e.g., midget impinger) to $\mathrm{PCM}$; c) job-exposure matrices used in the original cohort studies; d) matching of size distributions to epidemiologic studies. Another factor was developed to account for uncertainties in case ascertainment (mesothelioma, in particular, was often misdiagnosed in the past) and limitations due to how data were presented in the published summaries. These factors were combined into a single factor, which was then combined with the statistical uncertainty for the lung cancer and mesothelioma potency estimates $\left(\mathrm{K}_{\mathrm{L}} \mathrm{S}\right.$ and $\left.\mathrm{K}_{\mathrm{M}} \mathrm{s}\right)$ to arrive at "uncertainty bounds" for each potency value.

These uncertainty bounds were incorporated in a meta analysis that implemented an extension of the U.S. EPA lung cancer dose-response model (Eqs. 1 and 2) and mesothelioma dose-response model (linear in exposure metric defined by Eq. 8) that allowed for fibers of different types and in different size ranges to have unique potencies. Tests of the hypothesis that amphibole and chrysotile were equally potent in causing mesothelioma were firmly rejected (p-value $\leq 0.001)$ in four tests that incorporated different fiber widths in the exposure metric. The corresponding tests for lung cancer were mixed, with p-values ranging from 0.002 (significant) to 0.12 (not significant). The hypothesis that fibers between 5 and $10 \mu \mathrm{m}$ in length were equally potent as fibers longer than $10 \mu \mathrm{m}$, versus the alternative hypothesis that the longer fibers were more potent, were close to significant for lung cancer ( $\mathrm{p}$-values based on different fiber widths ranged from 0.05 to 0.12 ) but less so for mesothelioma (p-values ranged from 0.07 to 0.44 ).

This study illustrates a method for dealing with uncertainty in exposures in cohort studies, as well as the utility of meta analyses in resolving important issues in risk assessment. However, it was limited by the quality of the data available, particularly the data on the distributions of fiber sizes in the different cohorts. It is hoped that better information can be obtained in the future through use of 
archived air samples and regeneration of asbestos dust clouds that are representative of exposures in certain cohorts.

\section{ISSUES ARISING FROM RELIANCE ON DATA IN PUBLISHED SUMMARIES}

Risk assessments often have to rely on summarized results from published reports of an epidemiologic study. In fact, rarely does a risk assessor have access to the detailed exposure and disease-outcome history for each member of an epidemiologic cohort. There are several issues, limitations, and concerns associated with using summarized data, often to the point that the published data cannot be the basis of any meaningful analysis.

A typical case is one in which the reported responses are categorized into $\mathrm{N}$ groups defined by non-overlapping ranges of one or more measures of or surrogates for exposure (e.g., average exposure intensity, duration of exposure, etc.). A common method for applying such data in a risk assessment is to first fit an exposure-response model (e.g., for relative risk, Eqs. 10 or 11) to such data using Poisson regression. Such an analysis requires summary values (e.g., means) for the exposures (and any other variables used in the model) in each cell of the categorization. The person-year weighted average exposure in a cell is the most appropriate such summary value. A common reporting problem is that no such summary values are provided in published material. This problem is especially serious for those categories that have open-ended definitions (e.g., "> 20 ppm-years"). Not only are average exposures in such categories particularly problematic to estimate from the published data, often these estimates are very influential to the modeling results. It would appear to be a simple matter to report such averages and this would greatly facilitate the use of summarized data in risk assessment.

In many reports of epidemiologic study results, such categorization is presented as a series of one-dimensional tables, each one showing, separately and independently, the responses observed in each group defined by one of the measures. For example there will be a table showing the responses observed in several groups defined by duration of exposure; there will be a separate table showing the responses in several groups defined by intensity of exposure; and so on. However, as discussed above and shown in the general form in Eq. (3), it is the integration of exposures over time, e.g., in its simplest form, the cumulative exposure, that is the appropriate metric for use in risk assessment. There is no way to estimate reliably such exposure metrics from a series of independent, one-dimensional tabulations.

Cross tabulation of exposure intensity and duration of exposure is a better, although still inadequate, summarization practice. Such cross-classifications allow some simple cumulative exposure calculations to be made. However, consider the hypothetical summarization of an epidemiologic study shown in Table 3. Even this simple example shows that the cumulative exposure groups that could be defined from such a cross-tabulation are not distinct from one another; only the groups on the diagonal of such tabulation do not overlap one another. If cumulative exposure is a suitable metric for assessing risk, then the groups defined by this cross-classification are not optimally defined.
Table 3. Hypothetical Epidemiological Study Cross Tabulation of Exposure Intensity and Duration of Exposure: Computed Possible Ranges of Cumulative Exposures (ppm-yrs) within Each Group

\begin{tabular}{|c|c|c|}
\hline \multirow{2}{*}{ Average Intensity of Exposure (ppm) } & \multicolumn{2}{|c|}{ Duration of Exposure (yrs) } \\
\cline { 2 - 3 } & $\mathbf{1 - 1 0}$ & $\mathbf{1 0 - 2 0}$ \\
\hline \hline $0.1-0.5$ & $0.1-5$ & $1-10$ \\
\hline $0.5-1.0$ & $0.5-10$ & $5-20$ \\
\hline
\end{tabular}

The exposure of each individual in an epidemiologic cohort changes over time. As that individual progresses through their exposure history, different increments of time (e.g., year) are associated with different values for an exposure variable such as cumulative exposure. Consequently, counts of observed responses and the corresponding expected responses ought to be categorized by the person-year rather than by the individual. If expected values for the number of disease cases are included in the tabulations, those expected values should be based on the number of person-years contributed (by every individual in the cohort) to each of the exposure metric categories. In such tabulation a given individual will likely contribute personyears to more than one category. Thus, it is important that tabular summaries of epidemiologic results report the number of person-years contributing to the values in each cell of the table. It is also important to realize that categorizations based on the final, end-of-follow-up value of the exposure metric for each individual are not appropriate for fitting a dose-response model.

In a cross tabulation as shown in Table $\mathbf{3}$, it is possible to determine which person-years from each individual should go in which cells of that table. But, a false distinction is made with respect to cumulative exposure; different personyears of observation that have the same value for cumulative exposure can contribute observed and expected counts of the disease to different cells. Thus, a summarization that includes a tabulation or categorization by cumulative exposure would be more useful for risk assessment than a summary that includes a two-dimensional cross tabulation by duration of exposure and average exposure intensity.

But even were such tabulation by cumulative exposure available, there are other associated limitations. First of all, the risk assessment would be restricted to use of the cumulative exposure metric used in that tabulation. There would be no opportunity to explore the effects of lag times, of windows of exposures, or of other, different weightings of prior exposures on risk (e.g., as implemented in Eq. 3). Moreover, the risk assessor is not able to explore the effects on a Poisson regression of using different cut-points in the tabulation. - a potentially important issue in Poisson regression as cited earlier. On the other hand, the risk assessor would not be able to pursue Cox regression at all. Effects of other potentially informative covariables (e.g., gender, ethnicity, calendar year, age) could not be explored. It would be highly problematic to attempt to use published summarized data to develop models that utilize pharmacokinetically defined dose metrics, or models that incorporate mechanistic details of disease progression. 
Because of such limitations, risk assessments should have available the unsummarized data from the underlying epidemiologic studies whenever possible.

\section{CONVERTING A RISK MEASURE FROM AN EPIDEMIOLOGIC STUDY TO ADDITIONAL RISK}

As mentioned previously, an analysis of an epidemiologic cohort will often present results in measures of risk (e.g., relative risks) that are not directly relevant to a risk assessment. A relative risk of two for a very rare disease has a very different societal effect than a relative risk of two for a common disease such as heart disease. Thus, a measure of the absolute increase in risk (e.g., additional risk above background) is more useful in a risk assessment than a measure of the increase in relative risk. For example, the U.S. EPA will most often be interested in estimating the increased risk associated with a constant lifetime exposure to a member of the general U.S. population. OSHA may be interested in estimating the risk to a worker who is exposed to some potentially toxic agent during working hours, for a working lifetime from ages 20 through 65 . In both of those instances, it is the lifetime increased risk associated with the exposure scenario of concern that must be determined. In these cases, the lifetime risk is the probability that, over an entire lifetime, an exposed individual will develop the disease of concern (or die from that disease if mortality is the endpoint of the analysis) minus the corresponding probability for an unexposed individual.

The method for determining such risks is known as the life-table approach [29] (details of this method are outlined in the Appendix). This technique requires estimates of the age-specific rates of disease (morbidity or mortality) for the condition of concern, as well as the all-cause background mortality rates for the population of interest in the absence of the exposure being evaluated. These all-cause rates typically are obtained from tabulated area-wide rates (e.g., rates for the U.S. population). If relative risk is the endpoint estimated from the epidemiologic data, then rates for the disease of interest must also be applied to convert from relative risk to additional risk (see Appendix).

The life-table approach uses the all-cause mortality rates to account for inter-current mortality which would remove an individual from the risk pool for the disease of interest. Background all-cause mortality rates should be modified to include the effect of the exposure (Appendix, Eq. A9). If mortality risk is being estimated, this modification consists of subtracting from the all-cause mortality rates the background mortality from the disease of interest, and adding back the modified rates predicted in the presence of the assumed exposure scenario. However, if one is estimating the lifetime risk of becoming sick, but not necessarily dying, from the disease of interest, the appropriate modification to the all-cause mortality rates is to subtract from these rates the background mortality rate from the disease of interest, but to add back the modified rates of disease morbidity (rather than mortality) predicted in the presence of the assumed exposure scenario. This replacement of mortality rates by morbidity rates is needed because when calculating the lifetime risk of becoming sick, but not necessarily dying, from the disease of interest, removal from the risk pool can be due to either death from some other cause or the occurrence of an incident case of the disease (whether or not the case results in death).

Since inter-current mortality is accounted for in the lifetable approach, theoretically there is no need to stop followup at some age, e.g., by estimating additional lifetime risk by, say, age 80 . However, in practice the calculation is implemented as a sum over a range of ages (Appendix Eq. A7), which must terminate as some age. Nevertheless, continuing the calculation to older ages (e.g., age 100) can give a better indication of the true "lifetime" risk, although the accuracy of such a calculation may be affected due to limited range of older ages (e.g., > 85 years) for which agespecific rates are available. Experimentation with different formulae for calculating lifetime risk (e.g., Appendix, Eq. A4 and A5) and different upper age limits for the calculation can provide a sense of the relative contribution of the oldest ages to the risk estimate.

The life-table approach utilizes estimates derived from the analysis of the epidemiologic data under consideration, for example a relative risk estimate as a function of cumulative exposure. As discussed earlier, this typically involves values for certain parameters that are part of a function relating exposure to risk. An important requirement, sometimes overlooked when applying the life-table approach in practice, is to use the same functional form and the same exposure metric for the calculation of lifetime risk as was used in the analysis of the epidemiologic data. If a linear relative risk model using cumulative exposure lagged 10 years was fit to the epidemiologic data, then that same functional form using that same exposure metric must be applied to the life-table calculations; the estimated model parameters from the epidemiologic data must be applied in that life-table calculation.

Even though the epidemiologic analysis will have been applied to a certain subpopulation (e.g., workers who are primarily exposed between the ages of roughly 20 and 65) there is an implicit assumption that the parameters derived from that analysis can be applied to the wider population for whom risk estimates are desired. In fact, it is most often the case that the relative risk is assumed to be the same at all ages (for a fixed value of the exposure metric) even when the estimation is based on a set of individuals of rather restricted age ranges. Of course, should any covariables, such as age, have been included in the analysis, so that that assumption need not apply, it is important to include the covariables (with the parameter estimates derived for them) in the lifetable calculations.

In the application of the life-table approach, one must specify an exposure scenario, e.g., age at first exposure and age at last exposure, and the (possibly age-dependent) exposure level during the exposure period. It should be noted that the effect of exposure will generally extend past the end of the exposure period. For example, if the exposure metric used in the epidemiologic analysis is cumulative exposure, any exposure, no matter how remote in the past, will have a constant and permanent effect at all ages subsequent to that exposure. For example, the effect on the disease probability at age 70 of exposure to $X$ ppm for 1 year between the ages of 20 and 21 is the same as the effect of exposure to $\mathrm{X} \mathrm{ppm}$ for one year between ages 60 and 61 . One should consider 
carefully the implications associated with any metric of interest.

\section{EXAMPLE: CONVERTING RELATIVE RISK TO ADDITIONAL LIFETIME RISK}

We illustrate issues involved in calculation of additional risk using results from a study by McDonald et al. of workers exposed to chrysotile asbestos at a textile plant located in Charleston, South Carolina [30]. There have been several more recent studies of exposures and health effect among workers in this plant based on additional follow-up of the cohort [31-33]. This older study is intentionally used to emphasize that our purpose is solely to illustrate methods and issues and the results are not intended as up-to-date, reliable estimates of risk from exposure to asbestos.

The leftmost five columns of Table 4 (reproduced from Berman and Crump [5]) contain data recorded by McDonald et al. on the dose-response for lung cancer in this cohort. The first column gives the ranges of exposures in units of million particles per cubic foot-years (mpfc-y), as measured by midget impinger. The second column gives the average exposures assumed by Berman and Crump for these ranges. Although the assumption of an average of $130 \mathrm{mpcf}-\mathrm{y}$ for the unbounded range of $>80 \mathrm{mpfc}^{-} \mathrm{y}$ is particularly problematic, no other data are provided by McDonald et al. to inform this assumption. The third column gives the corresponding mean concentrations in fibers/ml-years as measured by PCM, and were obtained by multiplying the values in the second column by 6 , a conversion factor derived from air samples collected side-by-side and analyzed by midget impinger and PCM. The fifth column contains the expected number of lung cancer deaths based on age-, race-, and calendar-year-specific death rates for South Carolina men. The sixth column contains the observed number of lung cancer deaths and the fourth column the corresponding standard mortality ratio $(\mathrm{SMR}=$ [observed deaths] $/$ [expected deaths] x 100). The seventh and eight columns show the predicted numbers of lung cancer deaths obtained by Berman and Crump [5] using Poisson regression to fit the linear relative risk models defined by, respectively, (Eq. 1) with no separate background parameter and (Eq. 2) containing the background parameter $\alpha$ that allows for the possibility the background lung cancer mortality rate in the cohort differed from that in South Carolina men. In the implementation of the Poisson regression it was assumed that the observed number of lung cancer deaths in a cell (Table 4, sixth column) had a Poisson distribution with mean $=$ [expected number (Table 4, fifth column)] $\mathrm{x}$ [RR (Eqs. 1 or 2)]. The parameters $\mathrm{K}_{\mathrm{L}}$ and $\alpha$ were estimated by maximizing the resulting loglikelihood [34] and confidence bounds for these parameters were computed by the profile likelihood method [35,36]. The accumulation of exposure involved a lag time of ten years as assumed in the USEPA model (Eqs. 1 and 2). However, rather than assigning exposures to person-years of observation and accounting for the change in a subject's exposure over time, it appears that a single exposure measure (cumulative exposure at time of death or end of follow-up) was assigned to each subject. As discussed earlier, this approach is not optimal.

The estimate of $\alpha=1.07$ is not significantly different from $\alpha=1.0$ ( $p$-value $=0.8$ ), and the fit is acceptable using either value ( $\mathrm{p}$-value $=0.88$ and 0.95 ). However the confidence intervals for the potency parameter, $\mathrm{K}_{\mathrm{L}}$, are wider with $\alpha$ estimated. Although McDonald et al. did not have data on smoking habits in the cohort, it is reasonable to assume that their lung cancer rates could differ from those of South Carolina men in general due to differences in smoking habits, which would cause $\alpha$ to deviate from 1.0.

Accordingly, we will illustrate the calculation of additional risk from these data using the formulae in the Appendix and the estimate of $\mathrm{K}_{\mathrm{L}}=0.010(\text { fiber } / \mathrm{ml}-\mathrm{y})^{-1}(90 \%$ CI: $0.0044,0.025)$ obtained with $\alpha$ estimated as 1.07 . The exposure scenarios considered will be constant occupational

Table 4. Fit of Relative Risk Model (Eq. 2) to Lung Cancer Mortality Data in a Cohort Exposed to Asbestos in a South Carolina Textile Mill [30] (from [5], Table B7)

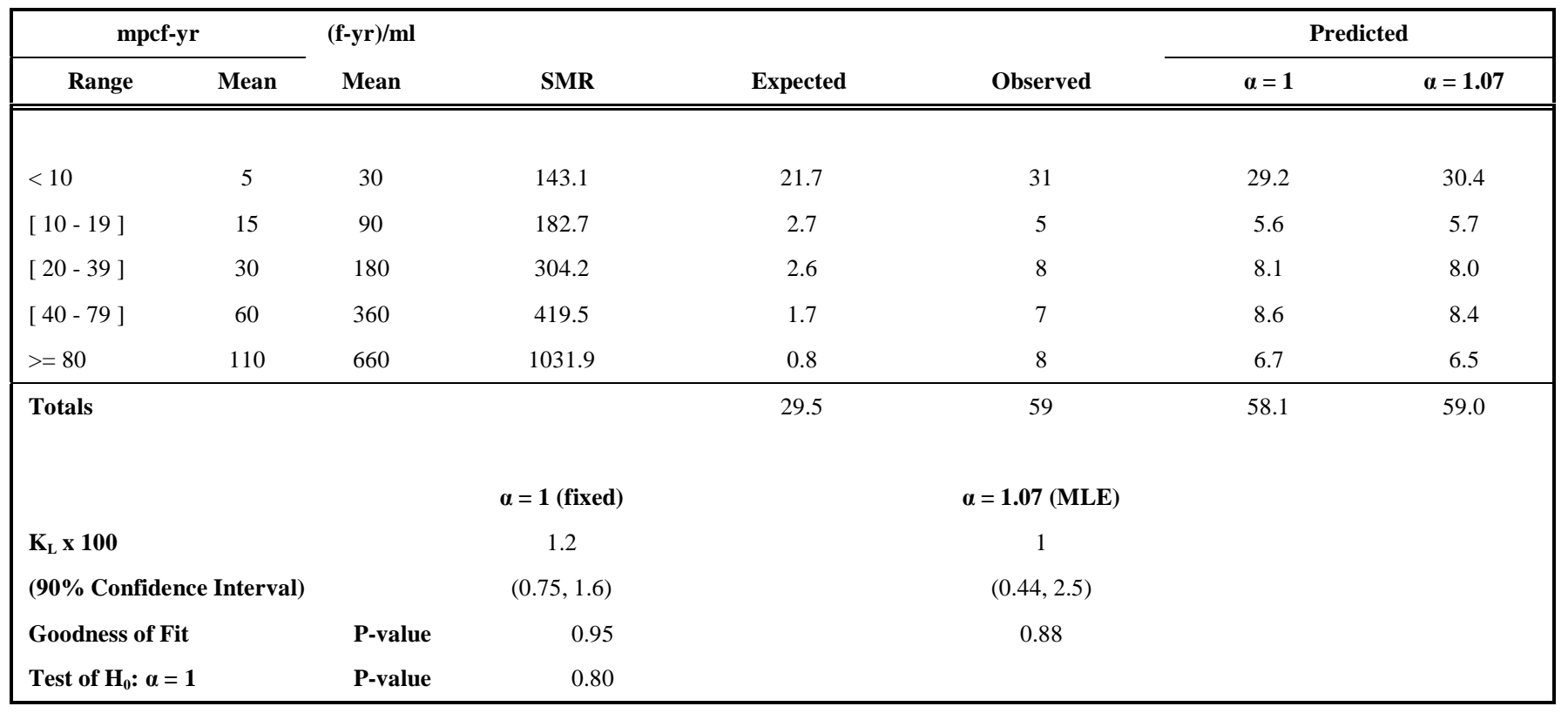


exposure from age 20 through age 65 to $\mathrm{E}$ fibers $/ \mathrm{ml}$. These exposure scenarios will be denoted by $\mathrm{D}_{\mathrm{E}}$, where $\mathrm{E}=0.001$ fibers $/ \mathrm{ml}$ or $1 \mathrm{fiber} / \mathrm{ml}$. The number of days per year exposed will be assumed to be the same as in the South Carolina cohort; otherwise $\mathrm{K}_{\mathrm{L}}$ could be adjusted to account for the difference.

To calculate the background risk of lung cancer mortality we apply Eqs. A6 and A7 in the Appendix. For age-specific death rates for all causes $\left(\mathrm{q}_{\mathrm{a}}(\mathrm{i})\right)$ and for lung cancer $\left(\mathrm{q}_{\mathrm{c}}(\mathrm{i})\right)$ we use the corresponding death rates in U.S. males and females combined for years 1985 through 1990. These rates are compiled by five-year age intervals, and the rate for a given five-year interval is used for each year in that interval. Likewise, the rates for the oldest age interval ( $\geq 85$ years) are used for all ages older than 85 years. Applying these rates in Eqs. A6 and A7 we obtain an estimate for the background probability $\mathrm{P}(0)$ of dying from lung cancer by age 85 $\left(x_{1}=20, x_{2}=85\right)$ of 0.047 and 0.053 with no limit on age $\left(\mathrm{x}_{1}=20, \mathrm{x}_{2}>=100\right)$.

As explained in the Appendix, estimating the probability of dying of cancer under one of these exposure scenarios uses the same formulae as in calculating $\mathrm{P}(0)$, only the rates $\mathrm{q}_{\mathrm{a}}(\mathrm{i})$ and $\mathrm{q}_{\mathrm{c}}(\mathrm{i})$ are replaced by modifications (Eqs. A8 and A9) that account for the effect of asbestos exposure from the assumed exposure scenario on these rates. For example, the term in the sum (Eq. A6) with $i=40$ corresponds to the year of age 40, during which the average age is 40.5 . Since the exposure metric used by McDonald et al. [30] was cumulative exposure lagged 10 years, the value of the exposure metric $\mathrm{D}_{\mathrm{E}}$ for this year is $(40.5-10-20) \mathrm{E}=10.5 \mathrm{E}$ fiber/ml-years. Consequently, using the linear relative risk model (Eq. 2) applied to the McDonald et al. data (Table 4), by (Eq. A8) $\mathrm{q}_{\mathrm{c}}(40)$ is replaced by $\mathrm{q}_{\mathrm{c}}(40)\left(1+10.5 \mathrm{EK}_{\mathrm{L}}\right)$ and by (Eq. A9) $\mathrm{q}_{\mathrm{a}}(40)$ is replaced by $\mathrm{q}_{\mathrm{a}}(40)+10.5 \mathrm{q}_{\mathrm{c}}(40) \mathrm{EK}_{\mathrm{L}}$. Notice that since the $\alpha$ value is specific to the South Carolina cohort, and the U.S. rates used in the calculation are assumed to be appropriate for the target population, these calculations do not involve $\alpha$. With these replacements $P\left(D_{E}\right)$ is calculated using Eq. A6 and A7 and the additional risk under exposure scenario $D_{E}$ is estimated as $\mathrm{P}\left(\mathrm{D}_{\mathrm{E}}\right)-\mathrm{P}(0)$. Upper or lower confidence bounds for additional risk can be adequately approximated using the same calculation except replacing the estimate of $\mathrm{K}_{\mathrm{L}}$ by its upper or lower confidence bound. An Excel spreadsheet for making these calculations can be obtained from the authors by request.

Table 5 shows the resulting estimates of additional risk of lung cancer death from occupational exposure from age 20 through age 65 to 0.001 fibers $/ \mathrm{ml}$ or 1 fiber $/ \mathrm{ml}$. Both the point estimates of risk and the confidence bounds increase in proportion to the exposure level in this range of exposures (although that would not continue to hold as the exposure level increased indefinitely). Estimates of lifetime risk (computed with $\mathrm{x}_{2} \geq 100$ years in Eq. A6) are about $12 \%$ larger than estimates terminated at age $80\left(\mathrm{x}_{2}=80\right)$. This illustrates that it is not necessary to posit a maximum age for the risk calculation when using the life-table approach.

\section{CONCLUDING REMARKS}

The focus of this paper has been on the application of data from cohort studies for the quantitative estimation of risk. Cohort studies are usually superior to other types of studies that may be available for a toxicant, at least as the basis for generating useful risk estimates. Cross sectional studies report the disease status of certain individuals who happen to be members of a specific subpopulation (e.g., workers in a particular industry) at a specific point in time. Such studies are plagued by difficulties related to timing: incident and prevalent cases cannot be differentiated. Therefore the exposures preceding the occurrence of the disease cannot be determined, and observation of members of the subpopulation at any particular time cannot account for drop-outs, who may very well have dropped out for reasons directly related to the exposure and/or the responses of interest. Case-control studies are often defined so as to avoid the some of the problems with cross sectional studies (e.g., there are no drop-out problems). But case-control studies seldom characterize exposure quantitatively (exposure is typically recorded as being present of absent). Moreover, without some specific design foresight (e.g., matching on age), there may be problems defining a relevant cumulative exposure metric for both the cases and the controls. There are also some technical issues related to using odds ratios estimated from case-control studies as estimators of relative risk that can be extrapolated to the general population of interest. However, when a more appropriate study is not available, a case-control study can possibly be used for a crude assessment of risk. In such a case, many of the issues discussed herein with regard to cohort studies would be applicable to a case-control study as well.

This paper has also focused on using data on binary endpoints (occurrence or non-occurrence of disease) to estimate the additional lifetime probability of disease from specified exposure patterns. Risk assessments can be developed for continuous endpoints, e.g., IQ decrements from exposure to methylmercury $[37,38]$. Methods similar to those discussed herein can be used to estimate useful measures of risk under specified exposure scenarios, e.g., the mean decrement in IQ or the addition fraction of individuals whose IQ falls below a certain value. Many of the issues faced in making such calculations are similar to those addressed herein.

Table 5. Effect of Extending to Older Ages the Calculation (Eq. A6) of Additional Risk of Lung Cancer Mortality from Occupational Exposure to Asbestos Fibers from Age 20 Through Age 65. Based on Data and Calculations Reported in Table 4

\begin{tabular}{|c|c|c|c|}
\hline Fibers/ml & $\begin{array}{c}\text { Risk by Age of } \\
\left(\mathbf{x}_{2} \text { in Eq. A6) }\right.\end{array}$ & Risk/1000 & $(\mathbf{9 0 \%}$ CI $)$ \\
\hline \hline 0.001 & 80 years & 0.017 & $(0.0072,0.041)$ \\
\hline & $\geq 100$ years $^{\mathrm{a}}$ & 0.020 & $(0.0084,0.047)$ \\
\hline 1.0 & 80 years & 17 & $(7.1,40)$ \\
\hline & $\geq 100$ years $^{\mathrm{a}}$ & 19 & $(8.3,46)$ \\
\hline
\end{tabular}

${ }^{a}$ Results remain the same to two significant figures for all ages $\geq 100$.

This paper has pointed out a number of potential limitations in a risk assessment that does not have access to the original data from an epidemiologic study, but is based only upon published summaries. We encourage risk 
assessors and epidemiologists to seek arrangements by which all of the unsummarized data can be made available for a risk assessment. For studies that are supported by public funds, we believe that once the original investigators have had a reasonable time to publish their findings, public access to the unsummarized data should be mandatory.

As discussed herein, the greatest uncertainties in quantitative risk assessments usually result from uncertainty in exposures in the epidemiologic studies. Going forward, industrial hygiene programs, air and food monitoring systems, etc. should be developed with the needs of future risk assessment in mind. For exposures that have already occurred, various methods can be applied to obtain better estimates of exposures in certain cases, such as recreating earlier exposure conditions. Nevertheless, uncertainty in exposure will continue to be a major limiting factor to the accuracy of risk estimates.

\section{ACKNOWLEDGEMENTS}

The work performed by KSC was supported by an appointment to the Research Participation Program of the National Center for Environmental Assessment, Environmental Protection Agency (EPA), through the Oak Ridge Institute for Science and Education (ORISE). Figs. $(\mathbf{1}, \mathbf{3})$ are used by permission of Informa Healthcare.

\section{APPENDIX}

\section{Formulae for Calculating Additional Risk}

The probability of disease occurrence (incidence or mortality) between ages $x_{1}$ and $x_{2}$ may be expressed as

$P(0)=\int_{x_{1}}^{x_{2}} h(x) S(x) d x$

where $\mathrm{S}(\mathrm{x})$ is the probability of survival to age $\mathrm{x}$ given survival to age $\mathrm{x}_{1}$ and $\mathrm{h}(\mathrm{x})$ is the instantaneous hazard of disease occurrence at age $x$. This integral can be approximated by a sum

$P(0)=\sum_{i=1}^{n} p(i) S(i)$,

where the age interval $\left[\mathrm{x}_{1}, \mathrm{x}_{2}\right]$ has been divided into $\mathrm{n}$ subintervals with the $\mathrm{i}^{\text {th }}$ subinterval having width $\Delta(i), \mathrm{i}=$ $1, \ldots, \mathrm{n}, \mathrm{p}(\mathrm{i})$, representing the probability of disease occurrence in the $i^{\text {th }}$ age interval, is calculated as

$\mathrm{p}(\mathrm{i})=\mathrm{q}_{\mathrm{c}}(\mathrm{i}) \Delta(\mathrm{i})$,

and $\mathrm{S}(\mathrm{i})$, representing is the probability of surviving to the beginning of the $i^{\text {th }}$ age interval, given survival to age $x_{1}$, is calculated as $S(1)=1$ and

$S(i)=\prod_{j=1}^{i-1} \exp \left[-q_{a}(i) \Delta(i)\right]=\exp \left[-\sum_{j=1}^{i-1} q_{a}(i) \Delta(i)\right], \quad i>1$

where $\mathrm{q}_{\mathrm{c}}(\mathrm{i})$ and $\mathrm{q}_{\mathrm{a}}(\mathrm{i})$ are the cause-specific rate of occurrence and all-cause death rates for the $\mathrm{i}^{\text {th }}$ age interval obtained from standard rate tables (29). An alternative to (Eq. A4) is given by

$$
S(i)=\prod_{j=1}^{i-1}\left[1-q_{a}(i) \Delta(i)\right], \quad i>1,
$$

which encompasses slightly different interpretations of the standard rates. These two expressions generally agree closely.

If the subintervals correspond to individual years, (Eq. A2 and A4) take on the simplified forms

$P_{0}=\sum_{i=x_{1}}^{x_{2}} q_{c}(i) S(i)$

and

$S(i)=\prod_{j=x_{1}}^{i-1} \exp \left[-q_{a}(i)\right]=\exp \left[-\sum_{j=x_{1}}^{i-1} q_{a}(i)\right]$

Once the background rates $\mathrm{q}_{\mathrm{c}}$ and $\mathrm{q}_{\mathrm{a}}$ are selected, these equations completely determine $\mathrm{P}(0)$. These same formulae are used to calculate the probability of response, $\mathrm{P}(\mathrm{D})$, from a particular exposure pattern, $\mathrm{D}$, by replacing the rates $\mathrm{q}_{\mathrm{c}}$ and $\mathrm{q}_{\mathrm{a}}$ by the appropriate modification that accounts for the model-predicted effect of exposure on these rates. The appropriate modifications depend upon the form of the doseresponse model estimated from the epidemiologic data, and the assumed exposure pattern. If the dose-response model predicts relative risk as a function of some exposure metric, then

$q_{c}(i)$ is replaced by $q_{c}(i) R(i)$,

(Eq. A8)

and

$q_{\mathrm{a}}(i)$ is replaced by

$q_{a}(i)-q_{c}(i)+R(i) q_{c}(i)=q_{a}(i)+q_{c}(i)[R(i)-1]$,

where $\mathrm{R}(\mathrm{i})$ is the relative risk predicted by the dose-response model at age $\mathrm{i}$ from exposure pattern $\mathrm{D}$. The latter replacement involves subtracting from the total death rate the background death rate from the disease of interest, and adding back this contribution adjusted by the effect of exposure. This expression assumes that mortality risk is being estimated. To estimate morbidity risk, the proper modification is to subtract from the total death rate the background death rate from the disease of interest, but add back the morbidity rate adjusted for the effect of exposure. This modification reflects the fact that when morbidity is the endpoint, a person is removed from the risk pool upon being identified as sick rather than when they die.

Once $\mathrm{P}(0)$ and $\mathrm{P}(\mathrm{D})$ have been calculated, the additional risk from exposure pattern $\mathrm{D}$ is computed as the difference

$P(D)-P(0)$

(Eq. A10)

These calculations are illustrated using a particular doseresponse model and exposure metric in the main part of the paper.

\section{REFERENCES}

[1] Cox DR. Regression models and life tables. R Stat Soc Ser B (methodol). 1972; 34: 187-220.

[2] Preston DL, Lubin JH, Pierce DA, McConney ME. Epicure User's Guide. Seattle: Hirosoft International Corporation 1988. 
[3] Voytek PE, Thorslund TW. Benzene risk assessment: status of quantifying the leukemogenic risk associated with the low dose inhalation of benzene. Risk Anal 1991; $11: 355-57$.

[4] Crump KS. Risk of benzene-induced leukemia: a sensitivity analysis of the pliofilm cohort with additional follow-up and new exposure estimates. J Toxicol Environ Health 1994; 42: 219-42.

[5] Berman DW, Crump KS. Update of potency factors for asbestosrelated lung cancer and mesothelioma. Crit Rev Toxicol 2008; 38: $1-47$.

[6] Armstrong BK, de Klerk NH, Musk AW, Hobbs MST. Mortality in miners and millers of crocidolite in Western Australia. Br J Indus Med 1988; 45: 5-13.

[7] de Klerk NH, Armstrong BK, Musk AW, Hobbs MST. Cancer mortality in relation to measures of occupational exposure to crocidolite at Wittenoom Gorge in Western Australia. Br J Indus Med 1989; 46: 529-36.

[8] de Klerk NH, Musk AW, Williams V, Filion PR, Whitaker D, Shilkin KB. Comparison of measures of exposure to asbestos in former crocidolite workers from Wittenoom gorge, W. Australia. Am J Indust Med 1996; 30: 579-87.

[9] Musk AW, de Klerk NH, Olsen NJ, et al. Mortality in miners and millers of crocidolite in Western Australia: Follow-up to 1999. Ann Occup Hyg 2002; 46, Suppl 1: 90-92.

[10] Rogers A, Neville M. Occupational and environmental mesotheliomas due to crocidolite mining activities in Wittenoom, Western Australia. Scand J Work Environ Health 1995; 21: 259-64.

[11] Rogers A, Major G. The quantitative risks of mesothelioma and lung cancer in relation to asbestos exposure: the Wittenoom data Ann Occup Hyg 2002; 46: 127-129.

[12] Berry G, de Klerk NH, Reid A, et al. Malignant pleural and peritoneal mesotheliomas in former miners and millers of crocidolite at Wittenoom, Western Australia. Occup Environ Med [Internet]. 2004; 61. Available from: (http://www.occenvmed.com/ cgi/content/full/61/4/e14)

[13] USEPA. Airborne Asbestos Health Assessment Update. Washington, D. C.: Office of Health and Environmental Assessment, US. Environmental Protection Agency, EPA/600/8/84/003F; 1986.

[14] Crump KS. Risk of benzene-induced leukemia predicted from the Pliofilm cohort with addtional follow-up and new exposure estimate. Environ Health Perspect 1996; 104 (Suppl) 6: 1437-41.

[15] Berman DW, Crump KS. A meta-analysis of asbestos-related cancer risk that addresses fiber size and mineral type. Crit Rev Toxicol 2008; 38: 49-73.

[16] Lutz WK, Lutz RW. Statistical model to estimate a threshold dose and its confidence limits for the analysis of sublinear dose-response relationships, exemplified for mutagenicity data. Mutat Res 2009; 678: 118-122.

[17] Steenland K, Mannetje A, Boffetta P, et al. Pooled exposureresponse and risk assessment for lung cancer in 10 cohorts of silica-exposed workers: an IARC multicentric study. Cancer Causes Control 2001; 12: 773-84.

[18] Steenland K, Deddens JA. A practical guide to dose-response analyses and risk assessment in occupational epidemiology. Epidemiol 2004; 15: 63-70.
[19] Berry G, Liddell FDK. The interaction of asbestos and smoking in lung cancer: A modified measure of effect. Ann Occup Hyg 2004; 48: 459-62.

[20] Hughes JM, Weill H, Checkoway H, et al. Radiographic evidence of silicosis risk in the diatomaceous earth industry. Am J Res Criti Care Med 1998; 158: 807-14.

[21] Buchanan BG, Miller BG, Soutar CA. Quantitative relations between exposure to respirable quartz and risk of silicosis. Occup Environ Med 2003; 60: 159-64.

[22] Moolgavkar SH, Dewanji A, Venzon DJ. A stochastic two-stage model for cancer risk assessment I. The hazard function and probability of cancer. Risk Anal 1988; 8: 383-92.

[23] Moolgavkar SH, Dewanji A, Luebeck G. Cigarette smoking and lung cancer: reanalysis of the British doctor's data. J Natl Cancer Inst 1989; 81: 415-21.

[24] Moolgavkar SH, Luebeck EG, Krewski D, Zielinski JM. Radon, cigarette smoke, and lung cancer: a re-analysis of the Colorado Plateau uranium miners' data. Epidemiology 1993; 4: 204-17.

[25] Moolgavkar SH, Luebeck EG, Buchmann A, Bock KW. Quantitative analysis of enzyme-altered liver foci in rats initiated with diethylnitrosamine and promoted with 2,3,7,8tetrachlorodibenzo-p-dioxin or 1,2,3,4,6,7,8-heptachlorodibenzo-pdioxin. Toxinol Appl Pharmacol 1996; 138: 31-42.

[26] Crump KS, Chen C, Chiu W, et al. What role for biologicallybased dose-response models in estimating low-dose risk? Environ Health Perspec 2010; 118: 585-88.

[27] Crump KS. The effect of random error in exposure measurement upon the shape of the exposure response. Dose Response 2005; 3: 456-64.

[28] Carroll RJ, Ruppert D, Stefanski LA. Measurement Error in Nonlinear Models. Boca Raton: Chapman \& Hall/CRC 2006.

[29] Gail M. Measuring the benefits of reduced exposure to environmental carcinogens. J Chronic Dis 1975; 28: 135-47.

[30] McDonald AD, Fry JS, Woolley AJ, McDonald JC. Dust exposure and mortality in an American chrysotile textile plant. Br J Ind Med 1983; 40: 361-367.

[31] Dement JM, Brown DP, Okun A. Follow-up study of chrysotile asbestos textile workers: cohort mortality and case-control analyses. Am J Ind Med. 1994; 26(4): 431-47.

[32] Hein JM, Stayner LT, Lehman E, Dement JM. Follow-up study of chrysotile textile workers: cohort mortality and exposure-response. Occup Environ Med 2007; 64: 616-25.

[33] Dement JM, Kuempel ED, Zumwaldt RD, Smith RJ, Stayner LT, Loomis D. Development of a fiber size-specific job-exposure matrix for airborne asbestos fibers. Occup Environ Med 2008; 65: 605-12.

[34] Cox D, Hinkley D. Theoretical Statistics. $1^{\text {st }}$ ed. Chapman \& Hall/CRC 1979.

[35] Venzon D, Moolgavkar S. A method for computing profilelikelihood-based confidence intervals. App Stats 1988; 37: 87-94.

[36] Crump KS. Benchmark Analysis. In: Encyclopedia of Environmetrics. West Sussex, U. K.: John Wiley \& Son 2002; 16370.

[37] National Research Council (NRC). Toxicological Effects of Mercury. Washington, DC: Nat Academies Press 2000.

[38] Clewell HJ, Crump KS. Quantitative estimates of risk for noncancer endpoints. Risk Anal 2005; 25: 285-289. 